\title{
Batches Algorithm for Passive Radar: A Theoretical Analysis
}

\author{
C. Moscardini, D. Petri, M. Conti, A. Capria, Member, IEEE, M. Martorella, Senior, Member, \\ IEEE, F. Berizzi, Senior, Member, IEEE
}

Abstract - Passive Bistatic Radar or Passive Coherent Location, is gaining interest in the radar community as it provides some advantages with respect to active radar. Passive radar does not aim to replace active radar, it provides a good complement to it. The computational effort that is required to implement the required signal processing is one of the drawbacks that affect passive radars. In this paper, a sub-optimal but computationally affordable algorithm is investigated that is applicable to arbitrary waveforms (different types of illuminator of opportunity) in order to achieve the target detection. Then a detailed mathematical formulation of the proposed sub-optimum algorithm and a theoretical performance analysis by comparing the proposed sub-optimum approach with the optimum 2D matched filter are provided. It is important to point out that the proposed theoretical analysis of the algorithm is not available in literature and it is independent of the type of waveform. Next, the analogies between the passive radar processing and the classical processing implemented in an active pulsed radar are underlined. Finally, simulated and real data are also used to demonstrate the effectiveness of the proposed algorithm and to validate the theoretical performance analysis.

Index Terms-Passive bistatic radar, passive coherent location, ambiguity function, illuminator of opportunity, range-Doppler map. 


\section{INTRODUCTION}

Passive Bistatic Radar (PBR) is gradually making progress both in terms of signal processing and system development (demonstrators and prototypes). Several e.m. sources, i.e. Illuminator of Opportunity (IO), can be used to implement such a radar system. Ground-based broadcast transmitters such as Frequency Modulation (FM) and Digital Audio Broadcasting (DAB) radio, as well as analogue and digital television have been largely exploited to demonstrate their capability to perform air and coastal surveillance [1].

In a PBR system, the optimum receiver (from the point of view of both detection and the parameters estimation theory) is implemented by calculating the so called two-dimensional Cross Ambiguity Function (CAF) [2], [3]. In other words, the CAF represents the optimum two dimensional matched filter that provides the signal processing gain to allow for the detection of targets and estimation of their bistatic range and Doppler shift. The evaluation of the CAF can be computationally expensive considering that large $2 \mathrm{D}$ range-Doppler maps might be required depending on the extent of the desired surveillance area and the radar resolution, both along the range and the Doppler coordinates. Moreover, a long integration time is usually required to achieve a desirable signal processing gain to allow for the detection of small and/or distant targets. Several sub-optimum algorithms have been proposed in the literature in order to achieve real time processing capabilities [2], [4], [5], [6], [7]. This paper will focus on one of these algorithms, namely the like-FMCW algorithm or batches algorithm, which was presented in [2] and in [7]. Specifically, in [2], a particular example of FM waveform has been presented, whereas in [7] a more analytical formulation has been proposed.

In [8] some preliminary results concerning the generalized formulation of the batches algorithm and the comparison in terms of computational load and SNR losses by using numerical simulations have been presented by the authors. Specifically, in this work a deep analytical development and a theoretical performance analysis of the algorithm have been defined. Moreover some parameters to evaluate the losses in terms of both peak amplitude and side lobe level of the batches algorithm with 
respect to the optimum algorithm have been defined. Finally the theoretical performance analysis has been validated by using both real and simulated data.

First of all, in Section II, a new revised formulation of the optimum CAF is proposed. After that, a new generalized and detailed mathematical formulation of the sub optimum batches algorithm is derived by considering a specific approximation in the Doppler domain. In Section III a theoretical comparative study between the optimum and the sub-optimum approach is presented in terms of Signal to Noise Ratio (SNR) and computational load. The theoretical analysis is carried out with the aim of predicting SNR losses when using the proposed algorithm in dependence of target and algorithm parameters. This analysis defines a useful procedure for estimating the performance of the batches algorithm. It is important to point out that the proposed theoretical analysis of the algorithm is independent of the type of waveform. In addition, the new proposed mathematical formulation of the revised optimum CAF processing and the batches algorithm suggest an interesting comparative analysis between the passive radar processing and the classical active pulsed radar processing. The analogies between the PBR processing and the classical active pulsed radar processing are discussed in Section III. Finally in Section IV, a case study that considers Digital Video BroadcastingTerrestrial (DVB-T) signals is presented and both simulated and real data are used to demonstrate the effectiveness of the proposed algorithm and to validate the theoretical performance analysis.

\section{REVISED FORMULATION OF THE BATCHES ALGORITHM}

\section{A. Problem Formulation}

In a passive radar system, the optimum way to process the received signal is given by the theoretical CAF calculation [2], [3] defined as

$$
\begin{gathered}
M(\tau, v)=\int_{0}^{T_{i n t}} x_{\text {surv }}(t) x_{r e f}{ }^{*}(t-\tau) e^{-i 2 \pi v t} d t \\
0 \leq \tau \leq \tau_{\max }-v_{\max } \leq v \leq v_{\max }
\end{gathered}
$$

where $M(\tau, v)$ represents the range-Doppler cross correlation function between the reference signal $x_{r e f}(t)$ 
and the surveillance signal $x_{\text {surv }}(t)$, the variable $\tau$ denotes the time delay, corresponding to the bistatic time of arrival difference, $\tau_{\max }$ is the maximum delay of interest and it is related to the maximum nonambiguous bistatic range, $v$ corresponds to the frequency Doppler shift, $v_{\max }$ is the maximum Doppler shift of interest and it is related to the maximum bistatic velocity of interest, $T_{\text {int }}$ denotes the integration time or the so called Coherent Processing Interval (CPI). The integration time is typically chosen equal to $T_{\mathrm{int}}=T_{o b s}+\tau_{\max }$, where $T_{o b s}$ is the length of the reference signal, in order to have no integration losses.

The most obvious way to view the cross correlation processing would be to calculate the Fourier Transform of the signal $x_{\text {surv }}(t) x_{r e f}{ }^{*}(t-\tau)$, known in literature as the mixing product, for each bistatic time delay. The second way to view the cross correlation processing would be to calculate the cross correlation between $x_{\text {surv }}(t)$ and $x_{r e f}(t-\tau)$ for each Doppler shift. These two approaches are known in literature as Cross Correlation and Fourier Transform methods respectively [2]. An efficient implementation of these approaches can be obtained by exploiting the well known FFT algorithm. Although the FFT approach can reduce the computational load of the CAF computation, there are several drawbacks of this approach and these must be underlined [2]. First of all, in the case of long integration time and with the high sampling frequency that may be required when using DAB, DVB-T, Worldwide Interoperability for Microwave Access (WiMAX) and Universal Mobile Telecommunications Systems (UMTS) types of signals, the FFT calculation may be impractical, as the sequences needing to be transformed would be too long. Secondly, the FFT algorithm takes into account all Doppler frequencies up to half of the sampling frequency. Typically, the maximum Doppler shift is much smaller than the sampling frequency (i.e. $v_{\max }<<f_{s}$ where $f_{s}$ is the sampling frequency). Therefore, the majority of the FFT points are discarded after being calculated.

The definition of sub-optimum implementations, in order to achieve real time processing capabilities, was discussed in the past by several authors [2-7]. The second issue may be resolved by applying a decimation technique that allows data to be discarded at Doppler frequencies where it is known that targets do not 
exist, before calculating the Fourier transform [6]. This paper will focus on one such algorithm, namely the like-FMCW algorithm or batches algorithm. A theoretical definition of the batches algorithm is proposed in the next section.

\section{B. Revised Formulation of the Optimum Theoretical CAF}

In this section a revised formulation of the optimum CAF, shown in equation (1), is proposed.

From equation (1), the reference signal $x_{r e f}(t)$ can be seen as the sum of $n_{B}$ contiguous batches of length $T_{B}$ and it can be written as

$x_{r e f}(t)=\sum_{k=0}^{n_{B}-1} x_{i}\left(t-k T_{B}\right)$

where $n_{B}$ is the number of batches obtained as

$n_{B}=\left\lfloor\frac{T_{o b s}}{T_{B}}\right\rfloor$

where $\lfloor$.$\rfloor indicates the maximum integer value that is smaller than the argument. It should be noted that$ $T_{o b s}$ is the length of the reference signal. The signal in each block is defined as

$x_{k}(t)=x_{r e f}\left(t+k T_{B}\right) q(t)$

where $q(t)$ is

$q(t)= \begin{cases}1 & t \in\left[0, T_{B}\right] \\ 0 & \text { otherwise }\end{cases}$

Substituting (2) into (1), the optimum CAF can be written as

$M(\tau, v)=\sum_{k=0}^{n_{B}-1} \int_{0}^{T_{\text {int }}} x_{\text {surv }}(t) x_{k}^{*}\left(t-\tau-k T_{B}\right) e^{-i 2 \pi v t} d t$

Starting from the following equation: 
$x_{k}^{*}\left(t-\tau-k T_{B}\right) \neq 0 \quad \forall t \in\left[k T_{B}+\tau, k T_{B}+\tau+T_{B}\right]$

the integral in Equation (6) can be modified as

$M(\tau, v)=\sum_{k=0}^{n_{B}-1} \int_{k T_{B}+\tau}^{k T_{B}+\tau+T_{B}} x_{\text {surv }}(t) x_{k}^{*}\left(t-\tau-k T_{B}\right) e^{-i 2 \pi v t} d t$

Then, with a change of variable $\alpha=t-k T_{B}$, it now follows that Equation (8) yields:

$M(\tau, v)=\sum_{k=0}^{n_{B}-1} e^{-i 2 \pi v k T_{B}} \int_{\tau}^{\tau+T_{B}} x_{\text {surv }}\left(\alpha+k T_{B}\right) x_{k}^{*}(\alpha-\tau) e^{-i 2 \pi v \alpha} d \alpha$

Because $\tau$ is supposed to be in the range 0 to $\tau_{\max }$, the integration limits, expressed in Equation (9), become 0 and $T_{B}+\tau_{\max }$. In addition, Equation (9) can be reformulated as

$M(\tau, v)=\sum_{k=0}^{n_{B}-1} e^{-i 2 \pi v k T_{B}} \int_{0}^{T_{B}+\tau_{\max }} x_{k}^{\text {surv }}(\alpha) x_{k}^{*}(\alpha-\tau) e^{-i 2 \pi v \alpha} d \alpha$

where $x_{k}^{\text {surv }}(t)$ is the k-th block of the surveillance signal, $x_{\text {surv }}(t)$, which is defined as

$x_{k}^{\text {surv }}(t)=x_{\text {surv }}\left(t+k T_{B}\right) g(t)$

where $g(t)$ is

$g(t)=\left\{\begin{array}{rr}1 & t \in\left[0, T_{B}+\tau_{\max }\right] \\ 0 & \text { otherwise }\end{array}\right.$

As it is possible to note from Equation (11) and Fig. 1, the blocks on the surveillance channel are partially overlapped. By considering the signals $x_{k}^{\text {surv }}(t)$ and $x_{k}(t)$, the CAF $M_{k}(\tau, v)$ (calculated within each batch) can be defined as

$M_{k}(\tau, v)=\int_{-\infty}^{+\infty} x_{k}^{s u r v}(t) x_{k}^{*}(t-\tau) e^{-i 2 \pi v t} d t$ 
$M(\tau, v)=\sum_{k=0}^{n_{B}-1} e^{-i 2 \pi v k T_{B}} M_{k}(\tau, v)$

$0 \leq \tau \leq \tau_{\max }-v_{\max } \leq v \leq v_{\max }$

Therefore, the optimum cross ambiguity function $M(\tau, v)$, between $x_{\text {surv }}(t)$ and $x_{\text {ref }}(t)$, can be seen as a weighed sum of the cross ambiguity functions, $M_{k}(\tau, v)$, evaluated within each batch.

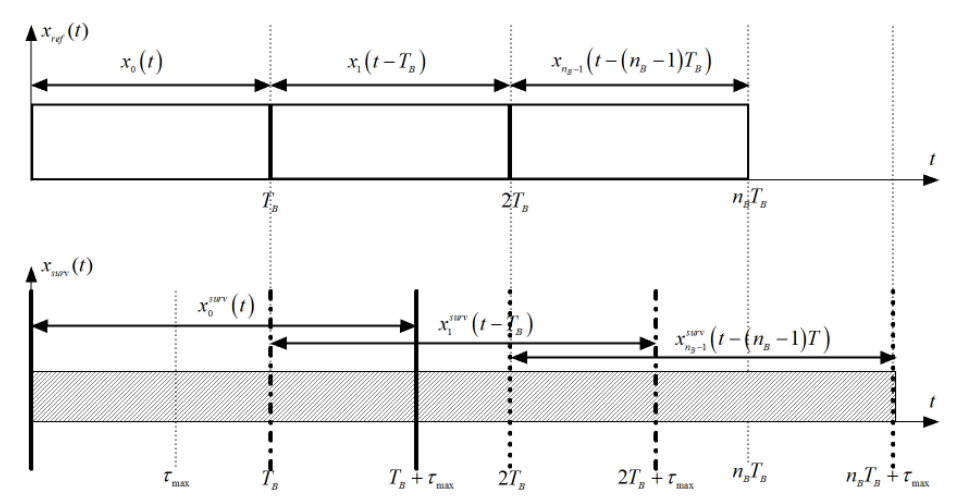

Fig. 1 Reference and surveillance signals segmentation

Up to this stage, only a new formulation of the optimum CAF has been suggested without gaining with respect to the computational load and time processing. Starting from equation (14), a description of the proposed sub-optimum method follows.

\section{Theoretical Definition of the Batches Algorithm}

In this section, a mathematical definition of the sub-optimum batches algorithm is derived by considering a specific approximation in the Doppler domain. Specifically, in (10), when the product between $T_{B}$ and the maximum target Doppler $v_{\max }$ is small compared to the unity, the phase rotation can be approximated within each block as a constant. Specifically, the value of such a phase rotation is closer to the central time of the batch interval $\left(T_{B} / 2\right)$

$$
2 \pi v t \approx 2 \pi v \frac{T_{B}}{2} \forall t \in\left[0, T_{B}\right], v \in\left[-v_{\max }, v_{\max }\right]
$$

and Equation (10) can be simplified as 
$M_{b}(\tau, v)=e^{-i \pi v T_{B}} \sum_{k=0}^{n_{B}-1} e^{-i 2 \pi v k T_{B}} \int_{0}^{T_{B}+\tau_{\max }} x_{k}^{\text {surv }}(\alpha) x_{k}^{*}(\alpha-\tau) d \alpha$

where the subscript "b" stands for "batches algorithm".

It should be noted that the target Doppler shift is estimated based on the increasing phased shift between consecutive batches (see Fig. 2). Due to the sampling theorem, this yields the relation $v_{\max } \leq 1 / 2 T_{B}$. In order to reduce the losses due to the small Doppler approximation given in equation (15) $T_{B} \leq 1 / 2 v_{\max } \gamma$ is considered, where $\gamma$ is a constant which could be defined in relation to an acceptable loss.

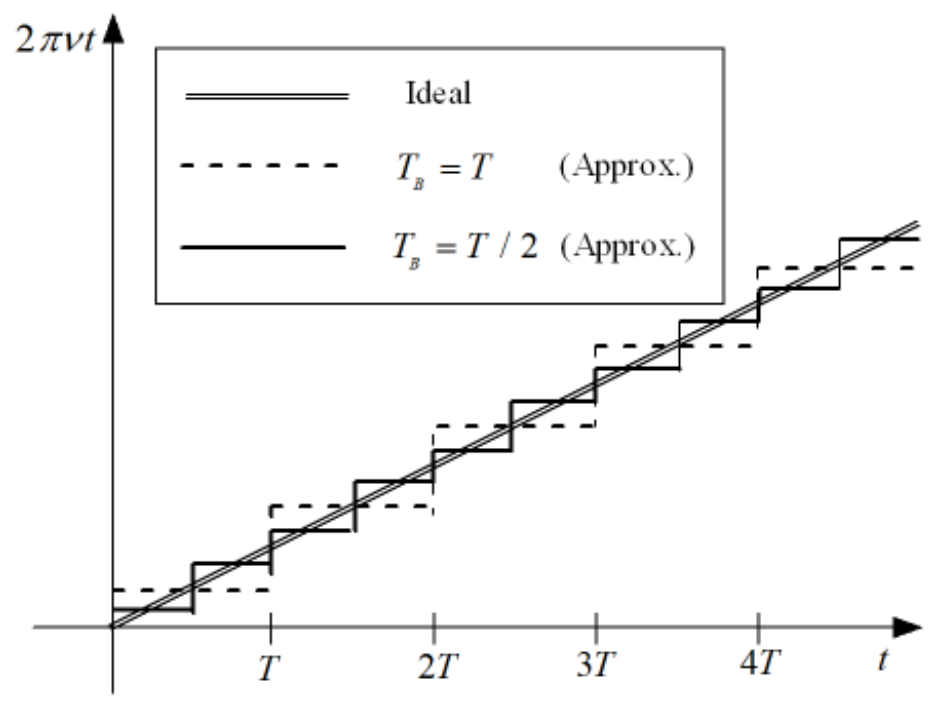

Fig. 2 Phase Approximation

Defining the cross correlation within the k-th block as

$x_{c c}^{k}(\tau)=\int_{0}^{T_{B}+\tau_{\max }} x_{k}^{\text {surv }}(\alpha) x_{k}^{*}(\alpha-\tau) d \alpha$

\section{equation (10) can be written as}

$M_{b}(\tau, v)=e^{-i 2 \pi v T_{B}} \sum_{k=0}^{n_{B}-1} e^{-i 2 \pi v k T_{B}} x_{c c}^{k}(\tau)$

or alternatively 
$M_{b}(\tau, v)=e^{-i 2 \pi v T_{B}} \sum_{k=0}^{n_{B}-1} e^{-i 2 \pi v k T_{B}} M_{k}(\tau, 0)$

The Doppler frequency compensation is neglected inside each block while it is considered with respect to consecutive blocks.

The main steps of this approach, schematically shown in Fig. 3, can be summarized as:

- Select $n_{B}$ consecutive batches $x_{k}(t)$ of the reference channel, and $n_{B}$ partially overlapped batches $x_{k}^{\text {surv }}(t)$ of the surveillance channel.

- Iteratively, for $\mathrm{k}=0, \ldots, n_{B}-1$ calculate the cross correlation, defined as $x_{c c}^{k}(\tau)$, between $x_{k}(t)$ and $x_{k}^{\text {surv }}(t)$ (see Equation (17))

- The Doppler dimension is then obtained by performing a Fourier Transform over the cross correlation values for each time delay, $\tau$, or range bin

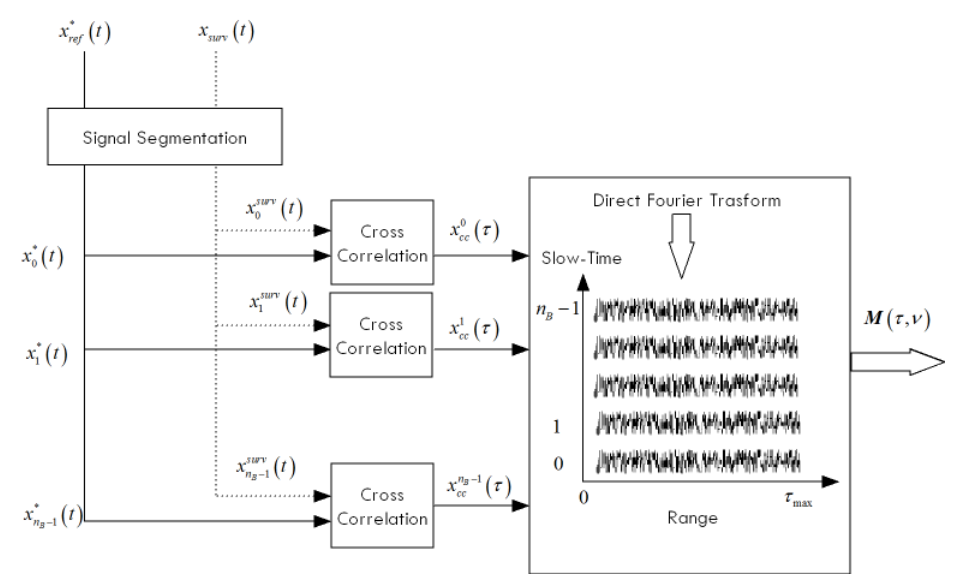

Fig. 3 Reference and surveillance signals segmentation

\section{THEORETICAL PERFORMANCE ANALYSIS - BATCHES ALGORITHM}

\section{A. SNR Analysis}

Typically, the radar performances are evaluated in terms of SNR. Under some typical assumptions, the 
received echo on the surveillance channel, after demodulation to baseband, can be well approximated by

$$
x_{\text {surv }}(t)=x_{\text {surv }}^{s}(t)+w(t)=A x_{\text {ref }}\left(t-\tau_{T}\right) e^{i 2 \pi v_{T} t}+w(t)
$$

where $\tau_{T}, v_{T}$ are the actual time delay and Doppler shift of a single slowly fluctuating point target. The complex constant A represents various effects like target reflectivity, propagation effects and antenna gain. $x_{r e f}(t)$ is the signal transmitted by the IO, $x_{\text {surv }}^{s}(t)=A x_{r e f}\left(t-\tau_{T}\right) e^{i 2 \pi v_{T} t}$ is the target received echo and $w(t)$ is an additive random noise process. The entire CAF calculation problem is linear, so the signal and the noise terms at the output can be separated as

$$
M(\tau, v)=M^{(s)}(\tau, v)+M^{(w)}(\tau, v)
$$

where $M^{(s)}(\tau, v)$ and $M^{(w)}(\tau, v)$ represent the useful signal and the noise component at the output of the CAF processing respectively.

Let us define the optimum SNR as the ratio of the signal power to the expected power in the noise term

$$
\operatorname{SNR}_{o p t}(\tau, v)=\frac{\left|M^{(s)}(\tau, v)\right|^{2}}{E\left\{\left|M^{(w)}(\tau, v)\right|^{2}\right\}}
$$

where $M^{(s)}(\tau, v)$ and $M^{(w)}(\tau, v)$ are respectively the target and noise components obtained at the output of the optimum CAF (shown in equation (14)) and $E\{$.$\} represents the statistical expectation operator.$

On the other hand, the signal to noise ratio $\operatorname{SNR}_{b}(\tau, v)$ at the output of the batches algorithm is calculated as

$$
\operatorname{SNR}_{b}(\tau, v)=\frac{\left|M_{b}^{(s)}(\tau, v)\right|^{2}}{E\left\{\left|M_{b}^{(w)}(\tau, v)\right|^{2}\right\}}
$$

where $M_{b}^{(s)}(\tau, v)$ and $M_{b}^{(w)}(\tau, v)$ represent the target and noise components obtained at the output of the batches algorithm respectively, as shown in equation (18). 
The target components $M^{(s)}(\tau, v)$ and $M_{b}^{(s)}(\tau, v)$, obtained respectively at the output of the optimum and batches algorithm, are calculated by substituting the target component $x_{\text {surv }}^{s}(t)$ into equations (14) and (18). This yields (see Appendix 1)

$$
\left\{\begin{array}{c}
M^{(s)}(\tau, v)=A e^{-i 2 \pi\left(v-v_{T}\right) \tau_{T}} \sum_{k=0}^{n_{B}-1} e^{-i 2 \pi\left(v-v_{T}\right) k T_{B}} \chi_{k}\left(\tau-\tau_{T}, v-v_{T}\right) \\
M_{b}^{(s)}(\tau, v)=A e^{i 2 \pi v_{T} \tau_{T}} \sum_{k=0}^{n_{B}-1} e^{-i 2 \pi\left(\nu-v_{T}\right) k T_{B}} \chi_{k}\left(\tau-\tau_{T},-v_{T}\right)
\end{array}\right.
$$

where $\chi_{k}(\tau, v)$ is the ambiguity function of the reference signal belonging to the $\mathrm{k}$-th batch, defined as

$$
\chi_{k}(\tau, v)=\int_{-\infty}^{+\infty} x_{k}(t) x_{k}^{*}(t-\tau) e^{-i 2 \pi v t} d t
$$

The noise components $M^{(w)}(\tau, v)$ and $M_{b}^{(w)}(\tau, v)$ obtained respectively at the output of the optimum and batches algorithm, are calculated by substituting the noise component $w(t)$ into equations (14) and (18). This yields (see Appendix 1)

$$
\left\{\begin{array}{c}
E\left\{\left|M^{(w)}(\tau, v)\right|^{2}\right\}=\sum_{k=0}^{n_{B}-1} \int S_{w}(f)\left|X_{k}(f-v)\right|^{2} d f \\
E\left\{\left|M_{b}^{(w)}(\tau, v)\right|^{2}\right\}=\sum_{k=0}^{n_{B}-1} \int S_{w}(f)\left|X_{k}(f)\right|^{2} d f
\end{array}\right.
$$

where $X_{k}(f)$ is the Fourier Transform of the reference signal $x_{k}(t)$ in the k-th batch and $S_{w}(f)$ is the noise power spectral density.

From equation (26) and under the assumption of additive white noise (AWN), the output noise power can be considered the same for both the batches and optimum algorithm. Assuming an additive coloured noise, the equality $\int S_{w}(f)\left|X_{k}(f-v)\right|^{2} d f=\int S_{w}(f)\left|X_{k}(f)\right|^{2} d f$ is a good approximation when the maximum considered Doppler $v_{\max }$ is small with respect to the band of the single batch transmitted signal. Under this assumption, the output noise power can be considered the same for both the batches and the optimum algorithm. 
Furthermore, to evaluate the losses of the batches algorithm with respect to the optimum algorithm, the Improvement Factor (IF) can be defined

$$
\operatorname{IF}(\tau, v)=20 \log _{10}\left(\left|\frac{S N R_{b}(\tau, v)}{S N R_{o p t}(\tau, v)}\right|\right)
$$

where $S N R_{b}(\tau, v)$ and $S N R_{o p t}(\tau, v)$ represent the SNR obtained respectively by using the batches algorithm and the optimum one.

From equation (27), the results obtained during the previous analysis equation yields

$$
\begin{aligned}
& I F(\tau, v)=20 * \log _{10}\left(\left|\frac{M_{b}^{(s)}(\tau, v)}{M^{(s)}(\tau, v)}\right|\right)=
\end{aligned}
$$

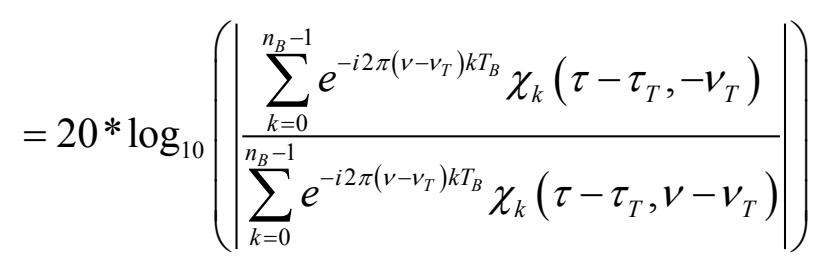

From equation (28) the ratio between the target peak evaluated with both the batches algorithm and the optimum one is

$$
\begin{aligned}
& \operatorname{PeakRatio}\left(\tau_{T}, v_{T}\right)=20 * \log _{10}\left(\left|\frac{M_{b}^{(s)}\left(\tau_{T}, v_{T}\right)}{M^{(s)}\left(\tau_{T}, v_{T}\right)}\right|\right)= \\
& =20 * \log _{10}\left(\left|\frac{\sum_{k=0}^{n_{B}-1} \chi_{k}\left(0,-v_{T}\right)}{\sum_{k=0}^{n_{B}-1} \chi_{k}(0,0)}\right|\right)
\end{aligned}
$$

In conclusion, the losses only depend on the target Doppler frequency $v_{T}$ and on the shape of the ambiguity function $\chi_{k}(0, v)$, evaluated within each batch, along the Doppler coordinate.

Specifically, if the small Doppler approximation in equation (15) can be accepted, the losses can be considered negligible. Moreover, the small Doppler approximation is acceptable if the product $v_{\max } T_{B}$ is small and for this reason, the batch length $T_{B}$ can be shortened as required in order to obtain reduced losses. It should be mentioned that the performance will depend on the type of waveform, as some 
waveforms are intrinsically less influenced by the Doppler effect than others. Therefore, the optimal selection of the batch length depends, in general, on the type of waveform. It is worth noting that equation (28) can be used to predict the performance of the algorithm with respect to the exploited IO and the target velocity. Specially equation (28) can be evaluated by using a closed-form expression of the ambiguity function $\chi_{k}(\tau, v)$, if it is available, or by computing $\chi_{k}(\tau, v)$ starting from the reference signal.

\section{B. Computational Cost Analysis}

An efficient implementation of lossless approaches, such as Correlation-FFT and Direct FFT, can be obtained by exploiting the well known Fast Fourier Transform (FFT) algorithm [2] as mentioned in section II-A. As discussed in [2], exploiting an FM illuminator of opportunity, and in [7],[8], where DVB-T signals were used, the processing time can be strongly reduced by using the batches algorithm. Specifically, in [8], it was shown that it is possible to reduce the processing time by more than $96 \%$ with respect to the optimum one. The computational load in terms of complex multiplication of the batches algorithm with respect to the two lossless approaches is shown in Table I. In particular, $N$ is the number of samples, $N_{b i n}$ and $N_{\text {Doppler }}$ represent the number of range and Doppler bins included in the CAF respectively. The computational load for the batches algorithm increases with the number of batches $n_{B}$ and the number $N_{b i n}$ of range bins included in the CAF. In other words, if the batch length $T_{B}$ decreases the number of batches $n_{B}$ increases and consequently the computational effort results more expensive due to the higher number of cross correlation computations.

TABLE I

Computational Cost

\begin{tabular}{ll}
\hline \hline Algorithm & Complex Multiplication \\
\hline Cross Correlation-FFT approach & $2 N \log _{2}(N)+\left[1+\log _{2}(N)\right] N N_{\text {Doppler }}$ \\
Fourier Transform-FFT approach & {$\left[1+\log _{2}(N)\right] N N_{\text {bin }}$}
\end{tabular}


Batch-Algorithm $\left[3 \log _{2}\left(N_{\text {bin }}\right)+1+\log _{2}\left(n_{B}\right)\right] N_{\text {bin }} n_{B}$

In conclusion the batch length $T_{B}$ can be set in order to obtain a good compromise between SNR losses and computational cost. Whilst the processing is not optimum, it is possible to obtain a real time CAF calculation with a general purpose PC, furthermore the losses should not significantly affect the detection performances setting the batch length in a correct way.

\section{Analogy with Pulse-Doppler Radar}

It is important to point out how the optimal theoretical CAF and the batches algorithm present analogies with the classical processing used in active pulse-Doppler radars. The common method typically used to process the received signal in an active pulse-Doppler radar is the pulse-Doppler processing [9]. The received signal is first pulse-compressed by correlating the fast-time signals with the zero-Doppler matched filter. The range-Doppler map is then formed by taking the Fourier Transform along the slow time domain. This processing is optimum working on the assumption that the received waveform is an amplitude-scaled and time-delayed version of the transmit waveform. The Doppler shift of the received waveform represents an unintentional mismatch between the received signal and the matched filter. The response of a waveform in the presence of an uncompensated Doppler is known in literature as "Doppler tolerance". In an active radar system, the Doppler shift and its impact on the matched filter output signal may be approached in several ways. The first way is to select a waveform and its modulation parameters in order to achieve a Dopplertolerant waveform. Secondly, if it is possible to estimate the target's radial velocity, the received signal can be cantered at baseband by applying an equal Doppler frequency shift to the received signal. Finally, a bank of matched filters, each one tuned to a different Doppler shift, may be used. This method can be considered as the optimum receiver in an active pulse-Doppler radar. PulseDoppler processing is perhaps the most commonly used method because it can often provide a 
significant reduction in computational cost while suffering only a modest loss in processing gain.

Comparing these two mentioned approaches with the optimal theoretical CAF and the batches algorithm, it is possible to conclude that there are the following analogies between the CAF calculation in a passive radar system and the typical processing adopted in active radar systems: the pulse repetition interval $T_{R}$ is the equivalent of the batch length $T_{B}$, the transmitted signal for each pulse $z(t)$ is the equivalent of the reference signal belonging to each batch $x_{k}(t)$ and the received signal $r_{k}(t)$ is the equivalent of $x_{k}^{\text {surv }}(t)$.

Specifically, the matched filter bank approach in an active pulse-Doppler radar, which implements a matched filter in both time delay and Doppler, is based on the same theoretical concept of the optimum CAF approach in a passive scenario. Both the pulse-Doppler and batches algorithm have been developed to reduce the computational load and their performances are strictly related to Doppler tolerance of the transmitted waveform.

Similarly to the passive radar scenario, the radar performances are evaluated in terms of SNR and the performances between the pulse-Doppler processing and the bank of matched filters are compared. Therefore, as shown in the case of passive radars, the following parameter is defined

$$
I F_{P D}(\tau, v)=20 \log _{10}\left(\left|\frac{S N R_{P D}(\tau, v)}{S N R_{M F}(\tau, v)}\right|\right)
$$

where $S N R_{P D}\left(\tau_{T}, v_{T}\right)$ and $S N R_{M F}\left(\tau_{T}, v_{T}\right)$ represent the $\mathrm{SNR}$ obtained by using the pulse-Doppler processing and the bank of matched filters, respectively.

Analogously to the passive radar analysis, under the assumption of AWN it can be shown that

$$
I F_{P D}(\tau, v)=20 * \log _{10}\left(\frac{\left|\chi_{Z}\left(\tau-\tau_{T},-v_{T}\right)\right|\left|\sum_{k=0}^{M-1} e^{-i 2 \pi\left(v-v_{T}\right) k T_{R}}\right|}{\left|\chi_{Z}\left(\tau-\tau_{T}, v-v_{T}\right)\right|\left|\sum_{k=0}^{M-1} e^{-i 2 \pi\left(v-v_{T}\right) k T_{R}}\right|}\right)
$$

where $\chi_{Z}(\tau, v)$ represents the ambiguity function of the transmitted pulse $z(t)$. 
Comparing equation (31) and equation (28) it is possible to conclude that the two expressions are very similar. In equation (28), by assuming $\chi_{k}(\tau, v)=\chi_{Z}(\tau, v) \forall i$, the same result is exactly obtained. The main difference is that, in the active radar case, the transmitted waveform properties can be selected in order to obtain a Doppler tolerant waveform, while in a passive radar scenario only the appropriate batch length can be selected to reduce the losses.

\section{CASE Study: DVB-T WAVEFORMS}

In this section, a specific case using DVB-T waveforms is considered . The theoretical performance analysis developed in Section III is evaluated via simulation and SNR losses are predicted in an operative scenario considering real data.

\section{A. Simulated Results}

Section III demonstrated how the losses of the target's echo power are negligible along the range coordinate. Therefore, in this section the losses with respect to both the batch length and the target's Doppler assuming a fixed target range are evaluated.

\section{1) Simulation set-up}

The DVB-T transmitted signal is simulated as

$$
x_{r e f}(t)=\sum_{k} \sum_{n=0}^{N-1} s_{O F D M}(k, t) q\left(t-k T_{S}\right)
$$

where $q(t)$ is defined as

$$
q(t)=\left\{\begin{array}{c}
1 \quad t \in\left[-T_{G}, T_{U}\right] \\
0 \text { otherwise }
\end{array}\right.
$$

In the previous equations, $T_{U}$ represents the symbol duration interval, $T_{G}$ represents the guard interval and $T_{S}=T_{G}+T_{U}$. In this multicarrier modulation scheme the $\mathrm{N}$ transmitted frequencies are orthogonal over a 
temporal window of length $T_{U}$. Considering the complex elements $s_{k, n}$ belonging to a finite alphabet and representing the transmitted digital data signal, the signal becomes

$$
S_{\text {OFDM }}(k, t)=\sum_{n=0}^{N-1} S_{k, n} e^{i 2 \pi \frac{n}{T_{U}} t}
$$

Next, results have been obtained by considering a $8 \mathrm{~K}$ DVB-T transmitted signal. The DVB-T signal standard is specified in [10], for convenience most parameters are reproduced in TABLE II.

TABLE II

DVB-T SigNAL SPECIFICATION

\begin{tabular}{ll}
\hline \hline Parameter & Values \\
\hline Subcarrier Spacing & $1.1 \mathrm{KHz}$ \\
Subcarriers Numbers & 6817 \\
Modulation & $64 \mathrm{QAM}$ \\
Bandwidth & $7.61 \mathrm{MHz}$ \\
Blocks per frame & 68 \\
OFDM symbol duration & $1.12 \mathrm{~ms}$ \\
Useful symbol duration & $T_{u}=896 \mu \mathrm{s}$ \\
Guard interval duration & $T_{u} / 4$ \\
\hline \hline
\end{tabular}

The received echo on the surveillance channel, after demodulation to baseband, is simulated according to equation (20). The other parameters used during the simulations are summarized in Table III.

TABLE III

SIMULATION PARAMETERS

\begin{tabular}{cc}
\hline \hline Parameter & Values \\
\hline Sampling Frequency & $f_{s}=9.14 \mathrm{MHz}$ \\
Observation Time & $T_{\text {obs }}=250 \mathrm{~ms}$ \\
Target Doppler Frequency & $v_{T}=[0,100,200,300,400,500,600,700,800,900] \mathrm{Hz}$ \\
Batch Time & $T_{B}=[15.64,31.28,109.38,218.76,333.29,462,924] \mu \mathrm{s}$ \\
\hline \hline
\end{tabular}

2) Results

As previously mentioned, the losses depend on the target Doppler frequency $v_{T}$ and on the shape of the ambiguity function $\chi_{k}(0, v)$, evaluated within each batch, with respect to the Doppler domain. 
For instance, in the case of a DVB-T signal, the zero range slice of the AF $\chi_{k}(0, v)$ can be viewed as a 'sinc' function $\operatorname{sinc}\left(T_{B} v\right)$, where $T_{B}$ is the batch length [7],[11]. In Fig. 4, the zero range slice $\left|\chi_{k}(0, v)\right|$, averaged on different batches, is calculated. The different lines refer to the considered batch lengths.

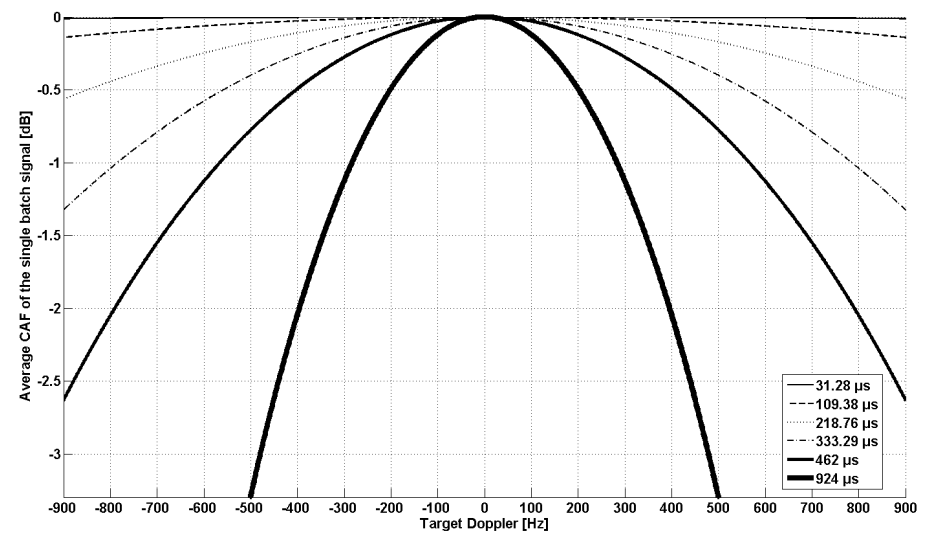

Fig. 4 Zero path delay AF of DVB-T signal

It is possible to conclude that, if the batch length is small, the zero path delay of the DVB-T ambiguity function is very flat and consequently the losses are very low even for large values of the target Doppler.

On the contrary, when the target's Doppler frequency and the batch length increase, the target's echo power decreases as shown in Fig. 5. Specifically, Fig. 5 shows the peak ratio defined in equation (29), for the considered target Doppler. Due to the sampling theorem the maximum target's Doppler is $v_{\max }=1 / 2 T_{B}$, as mentioned in Section II-B. For a batch length of $924 \mu \mathrm{s}$ the maximum target's Doppler is about $541 \mathrm{~Hz}$ and in Fig. 5 the line relative to this batch length is interrupted at the target's Doppler frequencies $>500 \mathrm{~Hz}$

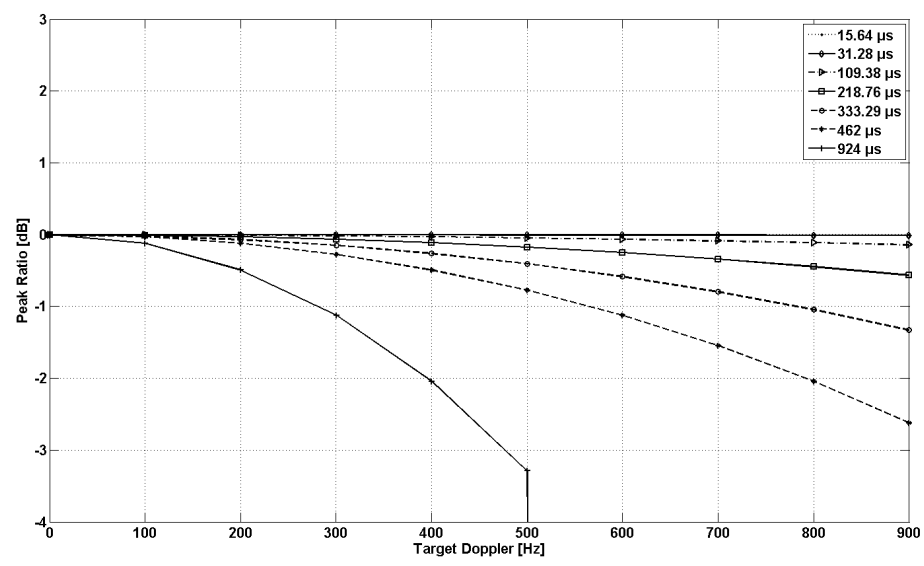




\section{Fig. 5 Peak ratio with respect to the target's Doppler}

From equation (28), the following metrics can be defined to evaluate the losses with respect to the CAF sidelobe levels:

- Integrated SideLobeLevel-Target's Doppler Profile: the ratio between the target's echo Doppler slice of the CAF evaluated with the batches algorithm and with the optimum approach

$$
I F\left(\tau, v_{T}\right)=20 * \log _{10}\left(\left|\frac{\sum_{k=0}^{n_{B}-1} \chi_{k}\left(\tau-\tau_{T},-v_{T}\right)}{\sum_{k=0}^{n_{B}-1} \chi_{k}\left(\tau-\tau_{T}, 0\right)}\right|\right)
$$

An estimate of the CAF background power is obtained by identifying a region $B(\tau)$ outside the main lobe of the target and by averaging the power in this region. The ratio between the normalized background power have been evaluated by using both the batches and optimum approach and have been defined as

$$
B P\left(\tau, v_{T}\right)=10 * \log _{10}\left(\frac{\int_{B(\tau)}\left|M_{b}^{(s)}\left(\tau, v_{T}\right)\right|^{2} d \tau}{\int_{B(\tau)}\left|M^{(s)}\left(\tau, v_{T}\right)\right|^{2} d \tau}\left|\frac{M^{(s)}\left(\tau_{T}, v_{T}\right)}{M_{b}^{(s)}\left(\tau_{T}, v_{T}\right)}\right|^{2}\right)
$$

- Integrated SideLobeLevel-Target's Range Profile: the ratio between the CAF range slices obtained with the batches and the optimum algorithm

$$
I F\left(\tau_{T}, v\right)=20 * \log _{10}\left(\left|\frac{\sum_{k=0}^{n_{B}-1} e^{-i 2 \pi\left(v-v_{T}\right) k T_{B}} \chi_{k}\left(0,-v_{T}\right)}{\sum_{k=0}^{n_{B}-1} e^{-i 2 \pi\left(v-v_{T}\right) k T_{B}} \chi_{k}\left(0, v-v_{T}\right)}\right|\right)
$$

Also in this case an estimate of the CAF background power is made by identifying a region $B(v)$ outside the main lobe of the target and by averaging the power in this region 


$$
B P\left(\tau_{T}, v\right)=10 * \log _{10}\left(\frac{\int_{B(v)}\left|M_{b}^{(s)}\left(\tau_{T}, v\right)\right|^{2} d v}{\int_{B(v)}\left|M^{(s)}\left(\tau_{T}, v\right)\right|^{2} d v}\left|\frac{M^{(s)}\left(\tau_{T}, v_{T}\right)}{M_{b}^{(s)}\left(\tau_{T}, v_{T}\right)}\right|^{2}\right)
$$

The results in terms of the two defined metrics (see equations (36) and (38)) are respectively shown in Fig. 6 and Fig. 7. It is possible to conclude that the losses with respect to the CAF background are negligible.

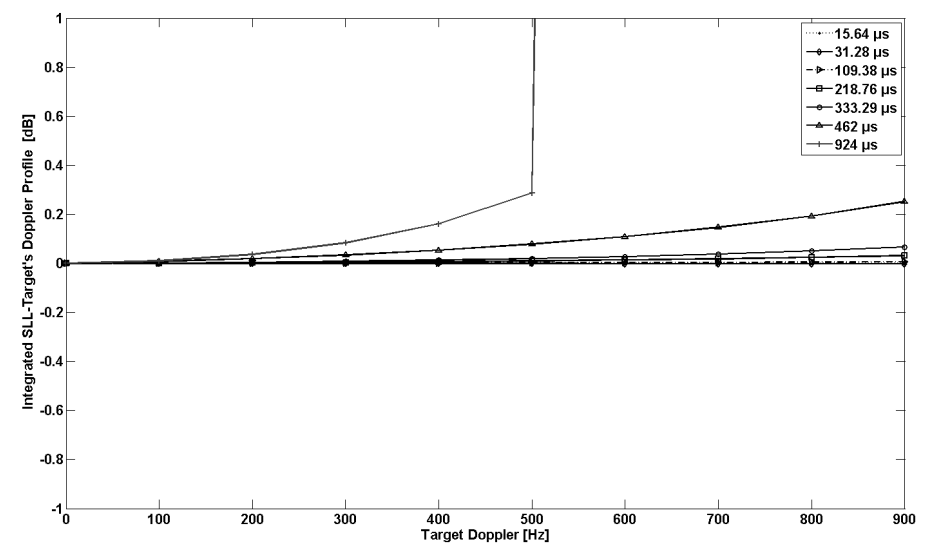

Fig. 6 SideLobeLevel-Target's Doppler Profile with respect to target's Doppler

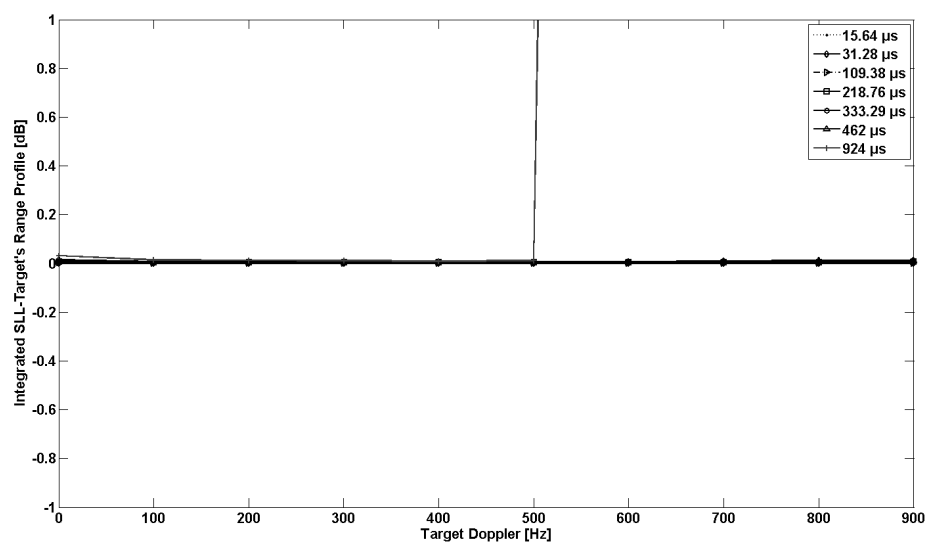

Fig. 7 Integrated SideLobeLevel-Target's Range Profile with respect to target's Doppler

The computational load in terms of complex multiplication is shown in Table IV. Specifically the computational load has been evaluated considering the theoretical analysis presented in Table I and the following observations: the number of Doppler bins $N_{\text {Doppler }}$ is equal to 250 considering an integration time of $250 \mathrm{~ms}$ and a maximum Doppler of $500 \mathrm{~Hz}$, the number of range bins is 1000 . The computational load 
of the batches algorithm with the largest batch length is reduced by a factor 18 or 72 with respect to the Cross Correlation-FFT approach and the Fourier Transform-FFT approach respectively.

TABLE IV

COMPUTATIONAL COST

\begin{tabular}{lc}
\hline \hline \multicolumn{1}{c}{ Algorithm } & Complex Multiplication \\
\hline Cross Correlation-FFT approach & $127 \cdot 10^{8}$ \\
Fourier Transform-FFT approach & $505 \cdot 10^{8}$ \\
Batch-Algorithm $\left(T_{B}=15.64 \mu \mathrm{s}\right)$ & $7.16 \cdot 10^{8}$ \\
Batch-Algorithm $\left(T_{B}=31.28 \mu \mathrm{s}\right)$ & $3.50 \cdot 10^{8}$ \\
Batch-Algorithm $\left(T_{B}=109.38 \mu \mathrm{s}\right)$ & $0.96 \cdot 10^{8}$ \\
Batch-Algorithm $\left(T_{B}=218.76 \mu \mathrm{s}\right)$ & $0.46 \cdot 10^{8}$ \\
Batch-Algorithm $\left(T_{B}=333.29 \mu \mathrm{s}\right)$ & $0.30 \cdot 10^{8}$ \\
Batch-Algorithm $\left(T_{B}=462 \mu \mathrm{s}\right)$ & $0.21 \cdot 10^{8}$ \\
Batch-Algorithm $\left(T_{B}=924 \mu \mathrm{s}\right)$ & $0.10 \cdot 10^{8}$ \\
\hline \hline
\end{tabular}

B. Real Data Results

1) Real data set-up

The experimental data were acquired in three different measurement campaigns:

1. The first campaign was supported by EDA in the context of the APIS (Array Passive ISAR) project [12], [13]. In particular, the acquisition system (RX) was located in Paracuellos near Madrid Barajas airport, whereas the DVB-T transmitter was located in Torrespana. Three DVB-T adjacent channels cantered at the frequency of $850 \mathrm{MHz}$ with a total bandwidth of about $25 \mathrm{MHz}$ are considered.

The integration time was $0.25 \mathrm{~s}$. The bistatic baseline was about $15 \mathrm{~km}$ and the target was a landing plane.

2. The receiver was located at the Department of Information Engineering of the University of Pisa and the used illuminator of opportunity was a DVB-T transmitter located on "Monte Serra" in Pisa [14]. Specifically the DVB-T transmitter was $14 \mathrm{~km}$ away from the receiver. The surveillance antenna was 
pointed at $17^{\circ}$ with respect to North (i.e.: azimuth) and tilted upwards towards the sky at an angle of $30^{\circ}$. The target was a plane.

3. The receiver was located at the "CSSN-ITE G. Vallauri" institute in Livorno and the used illuminator of opportunity was a DVB-T transmitter located on "Monte Serra" in Pisa (around $32 \mathrm{kms}$ from the receiver) and the surveillance antenna was directed towards an area of the sea in front of the receiver site Error! Reference source not found.. The targets were ships.

2) Results

Nine targets with different Doppler frequency values from the described data sets are selected. Specifically, the target types and their Doppler frequencies are summarized in Table V.

TABLE V

\begin{tabular}{cccc}
\multicolumn{4}{c}{ TARGETS PARAMETERS } \\
\hline \hline \multirow{2}{*}{ Data Set } & Target Type & Range & Doppler Frequency \\
\hline$\# 1$ & Plane & $2500 \mathrm{~m}$ & $-176 \mathrm{~Hz}$ \\
$\# 1$ & Plane & $3000 \mathrm{~m}$ & $268 \mathrm{~Hz}$ \\
$\# 1$ & Plane & $4000 \mathrm{~m}$ & $460 \mathrm{~Hz}$ \\
$\# 2$ & Plane & $1700 \mathrm{~m}$ & $-384 \mathrm{~Hz}$ \\
$\# 2$ & Plane & $950 \mathrm{~m}$ & $-344 \mathrm{~Hz}$ \\
$\# 3$ & Ship & $3700 \mathrm{~m}$ & $0 \mathrm{~Hz}$ \\
$\# 3$ & Ship & $7400 \mathrm{~m}$ & $40 \mathrm{~Hz}$ \\
$\# 3$ & Ship & $2500 \mathrm{~m}$ & $-24 \mathrm{~Hz}$ \\
$\# 3$ & Ship & $13000 \mathrm{~m}$ & $-36 \mathrm{~Hz}$ \\
\hline \hline
\end{tabular}

The comparison between simulations and real data is shown in Fig. 8. Specifically, the ratio between the target's signal peak with both the batches and the optimum algorithm is evaluated according to equation (29). Firstly, the peak ratio for the simulated signal (solid lines) by considering four batch lengths have been evaluated, then the peak ratio with respect to the real data (left-pointing triangle) have been calculated. It is important to point out that the values obtained with the real data closely match those obtained with simulation. The mismatch between simulation and real data amounts to a maximum absolute value of $0.5 \mathrm{~dB}$. This behaviour is due to the fact that the theoretical development has been obtained with the assumption of a slowly fluctuating target in AWN environment. In a real 
scenario, due to the presence of interference such as direct signal and clutter the assumption of AWN is not valid anymore.
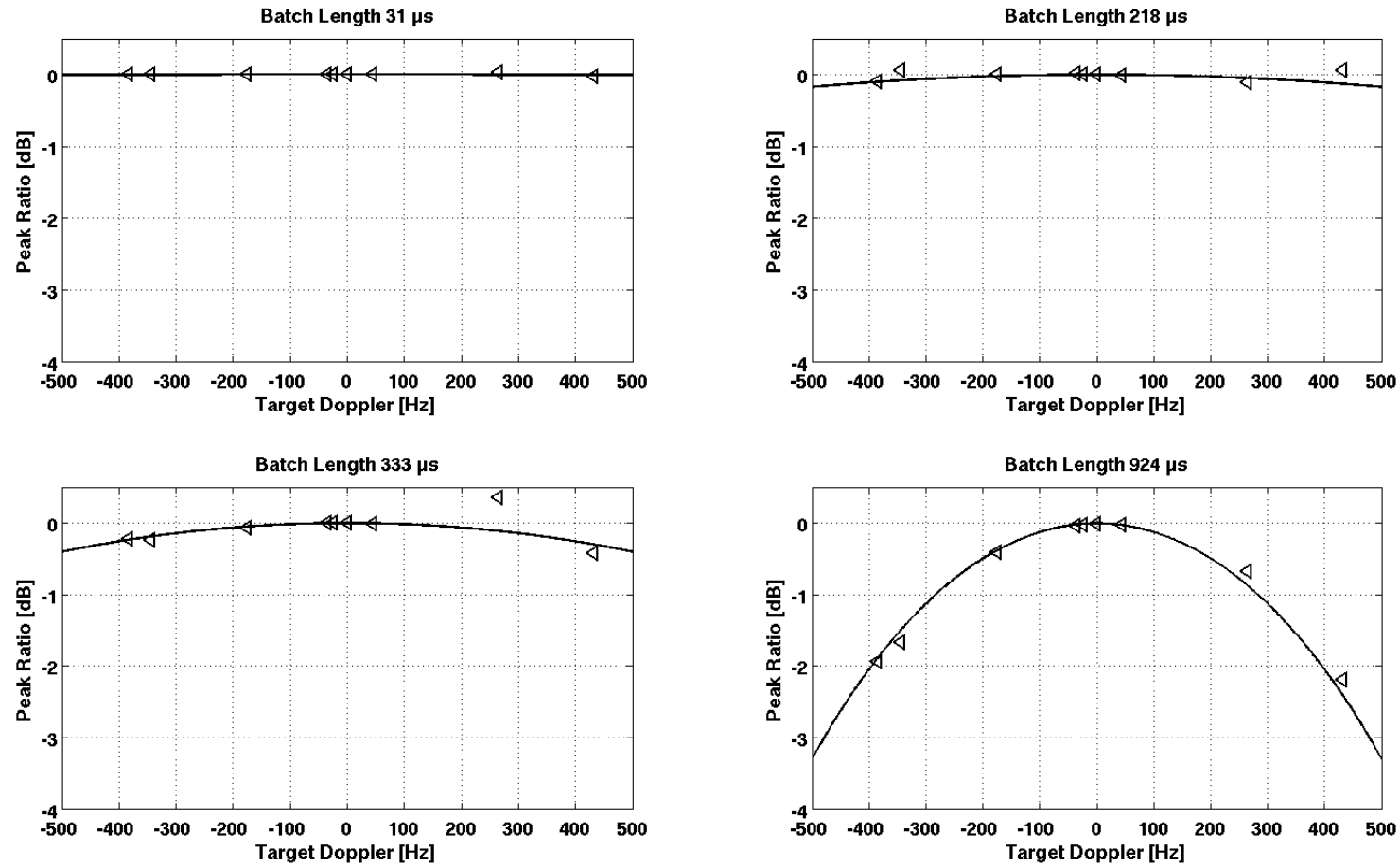

Fig. 8 Comparison between simulation and real data.

The results presented in Fig.8 are summarized in Table VI. The processing time has been evaluated with a general purpose 4 core PC equipped with 16 GB of RAM.

Specifically, as it is possible to notice from Fig.8, when the target Doppler frequency and the batch length increase the target power decreases. The processing time is related to the batch length, if the batch length $T_{B}$ decreases the computational effort results more expensive.

TABLE VI

SUMMARIZED RESULTS

\begin{tabular}{lccc}
\hline \hline Batch Length & $\begin{array}{c}\text { Peak Ratio } \\
v_{T}=-176 \mathrm{~Hz}\end{array}$ & $\begin{array}{c}\text { Peak Ratio } \\
v_{T}=460 \mathrm{~Hz}\end{array}$ & $\begin{array}{c}\text { Processing } \\
\text { Time }\end{array}$ \\
\hline$\left(T_{B}=31.28 \mu \mathrm{s}\right)$ & -0.0005 & -0.0303 & $4.93 \mathrm{~s}$ \\
$\left(T_{B}=218.76 \mu \mathrm{s}\right)$ & -0.0061 & 0.0536 & $0.92 \mathrm{~s}$ \\
$\left(T_{B}=333.29 \mu \mathrm{s}\right)$ & -0.0699 & -0.4297 & $0.71 \mathrm{~s}$ \\
$\left(T_{B}=924 \mu \mathrm{s}\right)$ & -0.4171 & -2.19 & $0.59 \mathrm{~s}$ \\
\hline \hline
\end{tabular}


These results demonstrate how the theoretical analysis can be used as a performance prediction tool.

Moreover, this analysis allows to set a batch length able to give a good compromise between losses and processing time with respect to the targets of interest and system requirements.

\section{CONCLUSion}

A novel formulation of the CAF processing for passive radar systems, namely, the batches algorithm has been proposed. A detailed mathematical analysis of the algorithm has been developed and a theoretical comparative study between the optimum and sub optimum algorithms has been presented in terms of SNR losses. An interesting comparative analysis between the proposed passive radar processing and the classical active pulsed radar processing has also been presented. Finally, the study has been supported by a comparison between the simulated and real data results, relatively to a DVB-T IO. The analysis of the results has confirmed the accuracy of the SNR losses predicted by the theory formulated.

\section{APPENDIX I}

The target components $M^{(s)}(\tau, v)$ and $M_{b}^{(s)}(\tau, v)$ obtained respectively at the output of the optimum and batches algorithm, are calculated starting from equation (14) and (18).

Firstly, by substituting the target component $x_{\text {surv }}^{s}(t)$, shown in equation (20), into equation (13), it can be shown that the target component of the $\mathrm{CAF} M_{k}^{(s)}(\tau, v)$, evaluated within each batch, is given by

$$
M_{k}^{(s)}(\tau, v)=\left\{\begin{array}{cc}
\sum_{m=1}^{M} \int_{0}^{\tau_{T}} x_{k-m}\left(t-\tau_{T}\right) x_{k}^{*}(t-\tau) e^{-i 2 \pi\left(v-v_{T}\right) t} d t+\chi_{k}\left(\tau-\tau_{T}, v-v_{T}\right) & 0 \leq \tau \leq \tau_{T} \\
\chi_{k}\left(\tau-\tau_{T}, v-v_{T}\right)+\sum_{l=1}^{L} \int_{T_{B}+\tau_{T}}^{T_{B}+\tau_{\max }} x_{k+l}\left(t-\tau_{T}\right) x_{k}^{*}(t-\tau) e^{-i 2 \pi\left(v-v_{T}\right) t} d t & \tau_{T} \leq \tau \leq \tau_{\max }
\end{array}\right.
$$

where $\chi_{k}(\tau, v)$ is the ambiguity function of the reference signal belonging to the $\mathrm{k}$-th batch, defined as

$$
\chi_{k}(\tau, v)=\int_{-\infty}^{+\infty} x_{k}(t) x_{k}^{*}(t-\tau) e^{-i 2 \pi v t} d t
$$


Secondly, by substituting the target component $x_{\text {surv }}^{s}(t)$, shown in equation (20), into equation (17), it can be shown that the target component of the cross correlation $x_{c c}^{k(s)}(\tau)$, evaluated within each batch, is given by

$$
x_{c c}^{k(s)}(\tau)=M_{k}^{(s)}(\tau, 0)=\left\{\begin{array}{cc}
\sum_{m=1}^{M} \int_{0}^{\tau_{T}} x_{k-m}\left(t-\tau_{T}\right) x_{k}^{*}(t-\tau) e^{i 2 \pi v_{T} t} d t+\chi_{k}\left(\tau-\tau_{T},-v_{T}\right) & 0 \leq \tau \leq \tau_{T} \\
\chi_{k}\left(\tau-\tau_{T},-v_{T}\right)+\sum_{l=1}^{L} \int_{T_{B}+\tau_{T}}^{T_{B}+\tau_{\max }} x_{k+l}\left(t-\tau_{T}\right) x_{k}^{*}(t-\tau) e^{i 2 \pi v_{T} t} d t & \tau_{T} \leq \tau \leq \tau_{\max }
\end{array}\right.
$$

where $\chi_{k}(\tau, v)$ is the ambiguity function of the reference signal belonging to the $\mathrm{k}$-th batch, defined in equation (40). It should be noted that in equations (39) and (41) the cross terms between two adjacent batches (see the sums with respect to $\mathrm{m}$ and 1) are equal to zero in the case $\left[0 \leq \tau \leq \tau_{T} ; k=0\right]$ and $\left[\tau_{T} \leq \tau \leq \tau_{\max } ; k=n_{B}\right]$. It is important to point out that the signals belonging to the two different batches can be considered uncorrelated, thus the cross terms can be ignored.

Under the previous assumption and by substituting equation (39) into equation (14), the target component $M^{(s)}(\tau, v)$ obtained at the output of the optimum CAF can be expressed as

$$
M^{(s)}(\tau, v)=e^{-i 2 \pi\left(v-v_{T}\right) \tau_{T}} \sum_{k=0}^{n_{B}-1} e^{-i 2 \pi\left(v-v_{T}\right) k T_{B}} \chi_{k}\left(\tau-\tau_{T}, v-v_{T}\right)
$$

where $\chi_{k}(\tau, v)$ is the ambiguity function of the reference signal belonging to the $\mathrm{k}$-th batch, as defined in equation (40).

On the other hand, starting from the same assumption and by substituting equation (41) into equation (18) it can be shown that the output target component $M_{b}^{(s)}(\tau, v)$ obtained with the batch algorithm is

$$
M_{b}^{(s)}(\tau, v)=e^{i 2 \pi v_{T} \tau_{T}} \sum_{k=0}^{n_{B}-1} e^{-i 2 \pi\left(v-v_{T}\right) k T_{B}} \chi_{k}\left(\tau-\tau_{T},-v_{T}\right)
$$

where $\chi_{k}(\tau, v)$ is the ambiguity function of the reference signal belonging to the $\mathrm{k}$-th batch, as defined in equation (40). 
After that, the noise components $M^{(w)}(\tau, v)$ and $M_{b}^{(w)}(\tau, v)$ obtained respectively at the output of the optimum and batches algorithm, are calculated starting from equation (14) and (18).

Firstly, by substituting the noise component $w(t)$, shown in equation (20), into equation (13), it can be shown that the noise component $M^{(w)}(\tau, v)$ is given by

$M^{(w)}(\tau, v)=\sum_{k=0}^{n_{B}-1} e^{-i 2 \pi v k T_{B}} M_{k}^{(w)}(\tau, v)$

where $M_{k}^{(w)}(\tau, v)$ is defined as

$M_{k}^{(w)}(\tau, v)=\int_{-\infty}^{+\infty} w_{k}^{\text {surv }}(\alpha) x_{k}^{*}(\alpha-\tau) e^{-i 2 \pi v \alpha} d \alpha$

and $w_{k}^{\text {surv }}(t)$ is

$w_{k}^{\text {surv }}(t)=w\left(t+k T_{B}\right) g(t)$

where $g(t)$ is

$g(t)=\left\{\begin{array}{rr}1 & t \in\left[0, T_{B}+\tau_{\max }\right] \\ 0 & \text { otherwise }\end{array}\right.$

The output noise power is

$$
\begin{aligned}
E\left\{\left|M^{(w)}(\tau, v)\right|^{2}\right\}= & \sum_{k=0}^{n_{B}-1} \sum_{l=0}^{n_{B}-1} e^{-i 2 \pi(k-l) v T_{B}} E\left\{M_{k}^{(w)}(\tau, v) M_{l}^{(w)^{*}}(\tau, v)\right\} \\
& =\sum_{k=0}^{n_{B}-1} E\left\{\left|M_{k}^{(w)}(\tau, v)\right|^{2}\right\}
\end{aligned}
$$

where

$E\left\{\left|M_{k}^{(w)}(\tau, v)\right|^{2}\right\}=\int S_{w}(f)\left|X_{k}(f-v)\right|^{2} d f$ 
and $E\{$.$\} represents the expectation operator and X_{k}(f)$ is the Fourier Transform of the reference signal $x_{k}(t)$ belonging to $\mathrm{k}$-th batch. It should be noted that equation (48) was obtained by assuming $E\left\{x_{k}^{(w)}(\tau) x_{l}^{(w)^{*}}(\tau)\right\}=0 \quad \forall l \neq k$

Whereas by replacing the noise component $w(t)$, shown in equation (20), with equation (18), the output noise component $M_{b}^{(w)}(\tau, v)$ obtained with the batch algorithm is

$$
M_{b}^{(w)}(\tau, v)=\sum_{k=0}^{n_{B}-1} e^{-i 2 \pi v k T_{B}} x_{c c}^{k(w)}(\tau)
$$

where the noise range profile $x_{c c}^{k(w)}(\tau)$ of the $\mathrm{k}$-th batch is defined as

$$
x_{c c}^{k(w)}(\tau)=\int_{-\infty}^{+\infty} w_{k}^{\text {surv }}(\alpha) x_{k}^{*}(\alpha-\tau) d \alpha
$$

and $w_{k}^{\text {surv }}(t)$ is defined in equation (46).

The power of the output sample evaluated over the range $\tau$ and for the $\mathrm{k}$-th batch is

$E\left\{\left|x_{c c}^{k(w)}(\tau)\right|^{2}\right\}=\int S_{w}(f)\left|X_{k}(f)\right|^{2} d f$

where $E\{$.$\} represents the expectation operator and X_{k}(f)$ is the Fourier Transform of the single batch reference signal $x_{k}(t)$.

From equation (50) and (52) and assuming $E\left\{x_{c c}^{k(w)}(\tau)\left(x_{c c}^{l(w)}(\tau)\right)^{*}\right\}=0 \quad \forall l \neq k$, the output power of the noise component is

$$
\begin{gathered}
E\left\{\left|M_{b}^{(w)}(\tau, v)\right|^{2}\right\}=\sum_{k=0}^{n_{B}-1} \sum_{l=0}^{n_{B}-1} e^{-i 2 \pi(k-l) v T_{B}} E\left\{x_{c c}^{k(w)}(\tau)\left(x_{c c}^{l(w)}(\tau)\right)^{*}\right\} \\
=\sum_{k=0}^{n_{B}-1} E\left\{\left|x_{k}^{(w)}(\tau)\right|^{2}\right\}
\end{gathered}
$$

ACKNOWLEDGMENT 
European Defence Agency (EDA) and funded by 11 contributing Members (Cyprus, France,

Germany, Greece, Hungary, Italy, Norway, Poland, Slovakia, Slovenia and Spain) in the framework of the Joint Investment Programme on Innovative Concepts and Emerging Technologies (JIP-ICET).

\section{REFERENCES}

[1] H. D. Griffiths, C. J. Baker, "Passive coherent location radar systems. Part 1: Performance prediction," IEE Proc. Radar, Sonar and Navigation, vol. 152, no. 3, pp.153-159, June 2005.

[2] P. E. Howland, H. D. Griffiths and C. J. Baker, "Passive Bistatic Radar Systems," in Bistatic Radar: Emerging Technology, Chapter 7, M. Cherniakov, John Wiley \& Sons, 2008.

[3] T. Tsao, M. Slamani, P. Varshney, D. Weiner, H. Schwarzlander, S. Borek, "Ambiguity function for a bistatic radar," IEEE Trans. Aerospace and Electronic Systems, vol.33, no.3, pp.1041,1051, July 1997.

[4] D. Langellotti, F. Colone, C. Bongioanni, P. Lombardo, "Comparative study of Ambiguity Function evaluation algorithms for Passive Radar,” in Proc. at the International Radar Symposium IRS 2009, Hamburg, Germany, Sep. 9-11, 2009.

[5] S. Stein, "Algorithms for ambiguity function processing," IEEE Transactions on Acoustics, Speech and Signal Processing, vol.29, no.3, pp. 588- 599, June 1981.

[6] P. E. Howland, et al, "FM radio based bistatic radar," IEE Proceedings Radar, Sonar and Navigation, vol.152, no.3, pp.107,115, 3 June 2005.

[7] J. Palmer, S. Palumbo, A. Summers, D. Merrett, S. Searle, S. Howard, “An overview of an illuminator of opportunity passive radar research project and its signal processing research directions," Digital Signal Processing, vol. 21, no. 5, pp. 593-599, September 2011.

[8] D. Petri, C. Moscardini, M. Martorella, M. Conti, A. Capria, F. Berizzi, "Performance analysis of the batches algorithm for range-Doppler map formation in Passive Bistatic Radar," in Proc. at the Radar Systems (Radar 2012) IET International Conference, 22-25 Oct. 2012. 
[9] M. A. Richards, J. A. Sheer, W. A. Holm, Principles of Modern Radar: Basic Principles, Lincoln Laboratory Journal, ShiTech Publishing, 2010.

[10] Digital Video Broadcasting (DVB), Framing Structure, Channel Coding and Modulation for Digital Terrestrial Television, ETSI std. EN300744 V1.6.1, January 2009.

[11]R. Saini, M. Cherniakov, "DTV signal ambiguity function analysis for radar application," IEE Proc. Radar, Sonar and Navigation, vol. 152, no. 3, pp.133-142, June 2005.

[12] J. Alvarez et al., “Array Passive ISAR Adaptive Processing (APIS) project: an overview," Specialist Meeting NATO SET-187 on "Passive Radar, Challenges Concerning Theory and Practice in Military Applications", Szczecin, Poland, 13-14 May 2013.

[13]D. Pasculli, A. Baruzzi, C. Moscardini, D. Petri, M. Conti, M. Martorella, "DVB-T Passive Radar Tracking on Real Data Using Extended Kalman Filter with DOA Estimation," in Proc. at the International Radar Symposium (IRS-2013), Dresden, Germany, June 19-21, 2013.

[14] M. Conti, F. Berizzi, M. Martorella, E. Dalle Mese, D. Petri, A. Capria, "High range resolution multichannel DVB-T passive radar," IEEE Magazine Aerospace and Electronic Systems, vol.27, no.10, pp.37,42, Oct. 2012.

[15] D. Olivadese, E. Giusti, D.Petri, M. Martorella, A. Capria, F. Berizzi, "Passive ISAR With DVBT Signals," IEEE Transactions on Geoscience and Remote Sensing, vol.51, no.8, pp.4508,4517, Aug. 2013

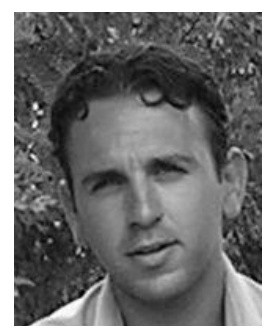

C. Moscardini was born in Castelnuovo Garfagnana, Lucca, Italy in December 1982. He received the Italian Laurea degree in Telecommunication Engineering (cum laude) in June 2008 from the University of Pisa (Italy). In March 2012 he received the Ph.D. degree in Remote Sensing at the University of Pisa. Since 2009 he has been involved as a researcher in several projects funded by Italian ministries and European organization (EDA). He is currently a Postdoc researcher at the Department of Information Engineering of the University of Pisa. His major research 
interests are in the field of passive radar, OTH radars, antenna array signal processing, Space Time Adaptive Processing and weather radar signal processing.

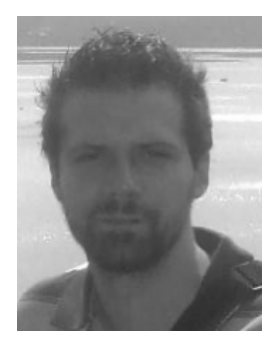

D. Petri received the Italian Laurea degree (M.S.) in Telecommunication Engineering in April 2007 from the University of Pisa, Italy. In March 2011 he received the Ph.D. in Remote Sensing at the University of Pisa. From 2011 to February 2013, he was with the RaSS Center where his major research interests were in the field of passive radar and synthetic range profile reconstruction. Since March 2013, he is a post doc researcher at the Department of Microelectronic at University of Delft (The Netherlands). He is now a member of the Microwave Sensing, Signals and Systems (MS3) group where he is working on Active and Passive Radar Networks.

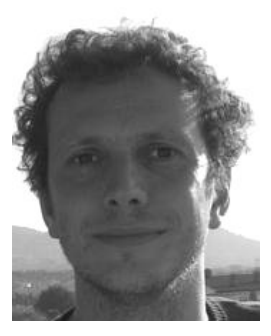

M. Conti was born in Livorno, Italy in March 1983. He received the Telecommunication Engineering Laurea and Ph.D. degrees from the University of Pisa (Italy) in 2009 and 2013, respectively. He is currently Post-doc researcher at Department of Information Engineering of the University of Pisa. Since 2009 Michele Conti has been involved in several scientific projects funded by Defense Ministry and European Defense Agency. His research interests are mainly in the field of passive radar system based on Software Defined Radio hardware.

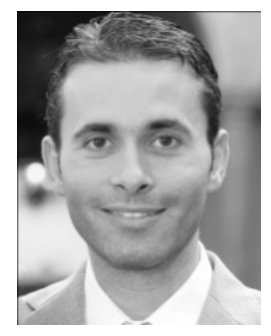

A. Capria was born in Pisa, Italy in June 1977. He received the Italian Laurea degree (M.S.) in Telecommunication Engineering in March 2004 from the University of Pisa, Italy. In March 2008 he received the Ph.D. in Remote Sensing at the Universty of Pisa. In April 2009 he became a CNIT researcher under contract and from April 2012 he is a CNIT 
permanent researcher. He has co-authored more than 50 papers and a book chapter. His major research interests are in the field of passive radar systems, OTH radars, antenna array processing, neural network applications in target classification, synthetic range profile reconstruction and design of fully digital radar based on photonic technologies.

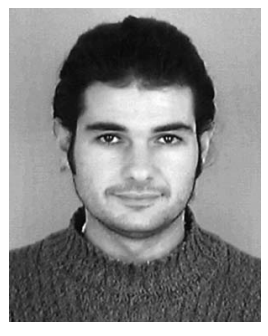

M. Martorella received his Laurea degree (Bachelor+Masters) in Telecommunication Engineering in 1999 (cum laude) and his PhD in Remote Sensing in 2003, both at the University of Pisa. He is now an Associate Professor at the Department of Information Engineering of the University of Pisa where he lectures "Fundamentals of Radar" and "Digital Communications" and an external Professor at the University of Cape Town where he lectures "High Resolution and Imaging Radar" within the "Masters in Radar and Electronic Defence". He is a regular visiting Professor at the University of Adelaide and at the University of Queensland in Australia. He is author of more than a hundred international journal and conference papers and three book chapters. He has presented several tutorials at international radar conferences and organised a special issue on Inverse Synthetic Aperture Radar for the Journal of Applied Signal Processing. He is a member of the IET Radar Sonar and Navigation Editorial Board, a senior member of the IEEE and a member of AFCEA. He is also chair of the NATO SET-196 on "Multichannel/Multistatic radar imaging of non-cooperative targets". He has been recipient of the 2008 Italy-Australia Award for young researchers, the 2010 Best Reviewer for the IEEE GRSL and the IEEE 2013 Fred Nathanson Memorial Radar Award. His research interests are mainly in the field of radar imaging, including passive, multichannel, multistatic and polarimetric radar imaging.

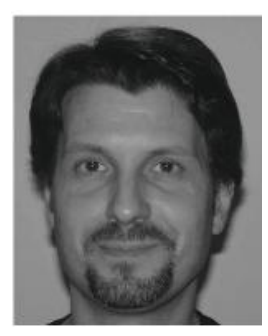

F. Berizzi (senior IEEE) received the Doctor Engineer and the Research Doctor degrees in electronic engineering from the University of Pisa, Italy, in 1990 and 1995 respectively 1990 and 1994 respectively.

He has been a full Professor of the Univ. of Pisa since Nov. 2009. He teaches "Radar techniques", "Signal Theory" at the Univ. of Pisa", and "Digital signal processing" at the Italian Navy 
Academic. He is also a visiting professor at the University of Cape Town where he lectures the course "High Resolution and Imaging Radar" within the International Masters in Radar and Electronic Defence. He is a IEEE Senior member since 2006.

He has been working on ISAR, SAR and radar systems since 1990. More than 150 papers and three chapters in international books have been published.

He has organised special and invited sessions at international conferences and workshops He has also given lectures and seminars in several research institutions in Australia and Europe and presented tutorials on SAR at IEEE Radar Conferences. He is the co-chair of the NATO Task Group on "Multichannel/Multistatic radar imaging of non-cooperative targets”, a member of four other NATO SET Task Groups.

Since 1992 Prof. Berizzi has been involved in several scientific projects as a Principal Investigator funded by the Ministry of Education, Ministry of Defence, Italian and European Space Agencies, Industries, Tuscany region, ESA (European Space Agency), European Defence Agency (EDA), NATO. 